\title{
A genetically informed study on the association of cannabis, alcohol, and tobacco smoking with suicide attempt
}

\author{
Massimiliano Orri $\mathbb{C}^{1,2}$ • Jean R. Séguin $\mathbb{1}^{3,4} \cdot$ Natalie Castellanos-Ryan $\mathbb{1}^{3,5} \cdot$ Richard E. Tremblay ${ }^{3,6,7}$.

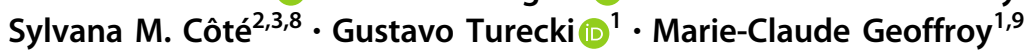

Received: 20 October 2019 / Revised: 6 May 2020 / Accepted: 12 May 2020

(c) The Author(s), under exclusive licence to Springer Nature Limited 2020

\begin{abstract}
Use of substances such as cannabis, alcohol, and tobacco, has been associated with increased risk of suicide attempt in several observational studies. However, establishing whether these associations are causal is challenging when using observational designs. To evaluate the potential causal contributions of cannabis use, alcohol use, and tobacco smoking to suicide attempt, we applied two-sample Mendelian randomization, an instrumental variable approach using single-nucleotide polymorphisms (SNPs) as instrumental variables for three exposures: lifetime cannabis use (yes/no; 42 instrument SNPs; GWAS sample size $[N]=162,082$ ), alcohol use (drinks-per-week; 53 instrument SNPs; $N=941,280$ ), and tobacco smoking (initiation, yes/no; 156 instrument SNPs; $N=1,232,091$; heaviness; 27 instrument SNPs; $N=337,334$ ). The main outcome was suicide attempt measured from hospital records $(N=50,264)$. All data come from publicly available summary statistics of genome-wide association studies of participants of European ancestry. We found evidence supporting a possible causal role of cannabis $(\mathrm{OR}=1.18 ; 95 \% \mathrm{CI}=1.01-1.37, P=0.032)$, alcohol $(\mathrm{OR}=1.95 ; 95 \% \mathrm{CI}=1.15-3.32, P=0.013)$, and smoking (initiation, $\mathrm{OR}=1.90 ; 95 \% \mathrm{CI}=1.54-2.34, P<0.001$; heaviness, $\mathrm{OR}=2.13 ; 95 \% \mathrm{CI}=1.13-3.99 ; P=0.019$ ) on suicide attempt. Using multivariable Mendelian randomization, we found that only cannabis showed a direct pathway to suicide attempt $(P=0.001)$, suggesting that the effect of alcohol and smoking was mediated by the other substance use phenotypes. No evidence was found for reverse causation, i.e., associations of suicide attempt on cannabis $(P=0.483)$, alcohol $(P=0.234)$, smoking initiation $(P=0.144)$, and heaviness $(P=0.601)$. In conclusion, evidence from this quasiexperimental study based on genetic data from large-scale GWASs are consistent with a causal role of cannabis, alcohol, and tobacco smoking on suicide attempt.
\end{abstract}

Supplementary information The online version of this article (https:// doi.org/10.1038/s41380-020-0785-6) contains supplementary material, which is available to authorized users.

Massimiliano Orri

massimiliano.orri@mail.mcgill.ca

1 McGill Group for Suicide Studies, Douglas Mental Health University Institute, Department of Psychiatry, McGill University, Montreal, QC, Canada

2 Bordeaux Population Health Research Centre, Inserm U1219, University of Bordeaux, Bordeaux, France

3 Sainte-Justine Hospital Research Center, Montréal, QC, Canada

4 Department of Psychiatry and Addictology, University of Montreal, Montreal, QC, Canada

\section{Introduction}

Suicide is an important public health concern worldwide, with $\sim 800,000$ people dying by suicide each year $[1,2]$. Suicide attempts, which are 20 times more frequent than suicide $[1,2]$, are strong predictors of suicide mortality.

5 School of Psychoeducation, Université de Montréal, Montreal, QC, Canada

6 School of Public Health, University College Dublin, Dublin, Ireland

7 Department of Pediatrics and Psychology, Université de Montréal, Montreal, QC, Canada

8 Department of Social and Preventive Medicine, School of Public Health, Université de Montréal, Montreal, QC, Canada

9 Department of Education and Counselling Psychology, McGill University, Montreal, QC, Canada 
They are also associated with unfavorable education, social, and health outcomes $[3,4]$. Prevention of suicide attempt is therefore a main public health concern.

Use of common substances such as cannabis, alcohol, and tobacco smoking [5] has been linked to increased suicide attempt risk in previous studies [6-14]. To date, most of the available evidence on the association of cannabis, alcohol, and tobacco smoking with suicide attempt comes from observational studies [6-14]. However, causality cannot be established from observational studies alone [15]. First, associations between substance use and suicide attempt may arise from confounding factors increasing the likelihood of both phenotypes. An example of such confounding factors is exposure to stressful life experiences, which can act as a common cause of both substance use and suicidal behavior [16]. Second, associations might be due to reverse causation. For example, previous studies showed that individuals reporting suicidal ideation/attempt were at increased risk of reporting substance use [17-19]. Indeed, suicidal manifestations may signal significant distress that individuals may self-medicate using substances [17-19]. Clarifying whether cannabis, alcohol, and tobacco smoking play a causal role on suicide attempt have therefore important public health implications [20]. Randomized trials would provide the strongest evidence of substance use's causal effect on suicide attempt. However, randomizing individuals into groups of substance use exposure would not be ethical.

Mendelian randomization, an instrumental variable approach relying on genetic data, can provide useful insights to clarify the possible causal effect of substance use on suicide attempt [21]. Mendelian randomization uses genetic variants (i.e., single-nucleotide polymorphisms; SNPs) robustly associated with an exposure (e.g., cannabis use) as proxy of the exposure to test its association with an outcome (e.g., suicide attempt; Fig. 1). Genetic variants are particularly suited to be used as instrumental variables because they are randomly transmitted at conception. Therefore, they are independent from the outcome and relatively independent from confounding of the exposureoutcome association [22]. Furthermore, associations estimated with Mendelian randomization are less prone than those from observational studies to be biased by reverse causation, because genotype is established at conception and does not change over the lifetime (see ref. [22] for a detailed account of strengths and limitations of Mendelian randomization). To our knowledge, no prior study used Mendelian randomization to strengthen causal inference on the role of cannabis, alcohol, and tobacco smoking on suicide attempt.

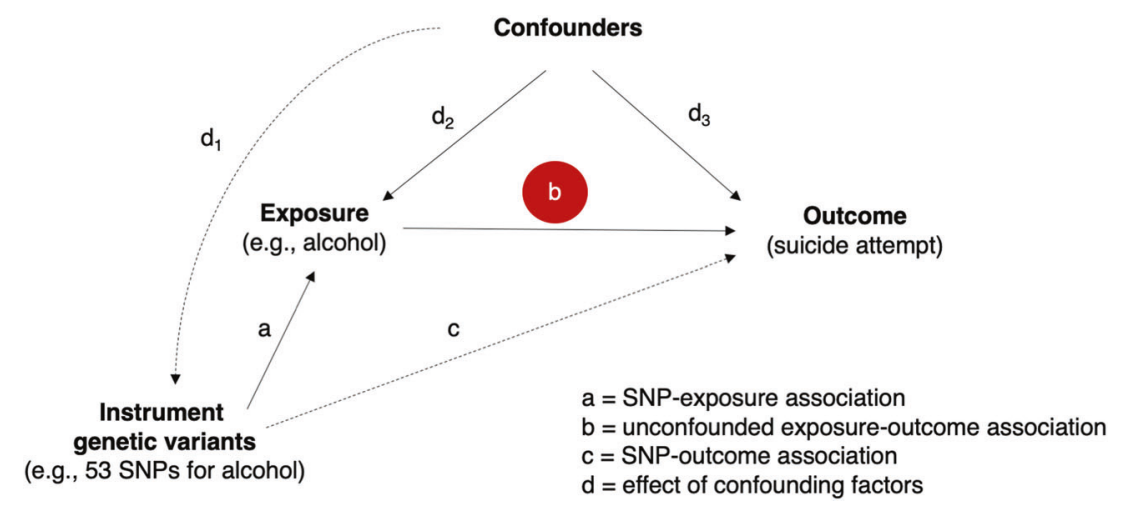

Fig. 1 Schematic representation of the Mendelian randomization design. The figure schematically represents the Mendelian randomization (MR) model. In two-sample MR, associations between the instrument genetic variants (SNPs) and the exposure come from a GWAS ( $a$ in the figure; e.g., the 53 SNPs identified as associated with alcohol in the alcohol GWAS), and associations between these same genetic variants and the outcome come from a different GWAS ( $c$ in the figure; e.g., the 53 previously selected SNPs were identified in the suicide attempt GWAS). This differs from the one-sample MR, where both the SNP-exposure and SNP-outcome associations (i.e., both $a$ and $c$ in the figure) come from the same GWAS. Different samples can be used in the same two-sample MR study, provided that all participants are from the same ancestry (e.g., European in this study) and that the two samples are not overlapping. The unconfounded association of the exposure (e.g., alcohol use) with the outcome (suicide attempt; $b$ in the figure) is estimated, for each SNP, as: $b=c / a$ (Wald test). The model relies on three main assumptions [21]. (1) Relevance: the instruments used must be robustly associated with the exposure, as assessed by a $F$ statistic $>10$ (e.g., the mean $F$ statistic for the 53 SNPs associated with alcohol was 80, range 28-964); (2) Exchangeability: the confounding factors of the exposure-outcome association, do not confound the SNPs-outcome association; i.e., $d_{1}$ is nonsignificant (e.g., stressfull life events can causally affect both alcohol use and suicide attempt, but cannot causally influence the SNPs inherited at conception); (3) Exclusion restriction criterion: there is no association between the instruments and the outcome conditional on the exposure, i.e., the only pathway of association of the instruments to the outcome must be through the exposure. This assumption may be violated by the horizontal pleiotropic effect of the SNPs used as instruments, which refers to the fact that SNPs can be associated with traits/genes that can potentially open alternative pathways through which the instruments may be associated with the outcome, and that are not in the causal pathway between the instruments and the outcome. Horizontal pleiotropy, if unbalanced, violates the exclusion restriction criterion, and methods such as MR-Egger should be used to account for pleiotropy. 
Relying on summary statistics from large genome-wide association studies (GWASs) performed by international consortia, the present study applied Mendelian randomization to test the potential causal role of cannabis, alcohol, and tobacco smoking on suicide attempt.

\section{Methods}

\section{Data and phenotype assessment}

\section{Data source}

This study is based on publicly available summary statistics of large GWASs with participants of European ancestry (Table 1). Only European ancestry was considered because of the lack of GWAS including participants of other ancestries for the phenotypes of interest.

\section{Assessment of suicide attempt}

Data came from the iPSYCH sample and consisted of data on individuals born in Denmark between May 1, 1981 and December 31, 2005 [23]. The sample consisted of 6024 cases with a record of suicide attempt and 44,240 controls with no record of a suicide attempt. In this sample, suicide attempt was derived from registry records.

\section{Assessment of cannabis use}

Data for lifetime cannabis use came from a meta-analysis GWAS using data from the International Cannabis Consortium (including 13 cohorts; $N=35,297$ ) and the UK Biobank (UKB; $N=126,785$ ) [24]. Data on whether the participants had ever used cannabis during their lifetime (yes/no) were available via self-reports.

\section{Assessment of alcohol use}

Data for alcohol use came from the GWAS and Sequencing Consortium of Alcohol and Nicotine use (GSCAN) meta-analysis, comprising samples from 26 cohorts $(N=$ $941,280)$ including UKB $(N=311,126)$ and 23 andMe $(N=403,931)$ [25]. The measured phenotype was drinksper-week, defined as the average number of drinks a participant reported drinking each week, aggregated across all types of alcohol.

\section{Assessment of tobacco smoking}

Data for tobacco smoking came from the GSCAN consortium [25]. Two phenotypes were considered: smoking initiation $(N=1,232,091$, including 383,631 participants 
from UKB and 599,289 participants from 23andMe), and smoking heaviness $(N=337,334$, including 120,744 form UKB and 73,380 from 23andMe). Smoking initiation was defined as ever having been a regular smoker (current or former), while smoking heaviness was defined as the average number of cigarettes smoked per day, either as current or former smokers. Both phenotypes were selfreported. Individuals who never smoked did not participate in the smoking heaviness GWAS.

\section{Two-sample Mendelian randomization analysis}

We performed a two-sample Mendelian randomization. In this design, two different GWAS summary statistics are used to obtain, respectively, the SNP-exposure and the SNP-outcome associations. The analysis was performed according to the following steps.

\section{Selection of instrument SNPs}

To determine the SNP-exposure associations, we selected, for each exposure phenotype GWAS (i.e., cannabis, alcohol, smoking initiation, and smoking heaviness), a set of instrument SNPs associated with the phenotype at the genome-wide level (i.e., $P<5 \times 10^{-8}$ ). When no or only few SNPs reached this threshold, SNPs at a suggestive statistical significance level $\left(P<1 \times 10^{-5}\right)$ were used $[24,26,27]$. As the method required the SNPs instruments to be independent, we performed linkage disequilibrium clumping to remove SNPs genetically correlated (i.e., window, $\left.10,000 \mathrm{~kb} ; r^{2}, 0.001\right)$. The strength of the final set of instruments was evaluated using the $F$ statistic, for which values above ten indicate strong instruments [28]. Subsequently, to determine the SNP-outcome associations, the selected SNPs were identified in the outcome (i.e., suicide attempt) GWAS.

\section{Association of cannabis, alcohol, and tobacco smoking (the exposures) with suicide attempt (the outcome)}

The following analyses were performed for each exposure. For each SNP instrument indexing the exposure, we calculated the Wald estimate. This is the ratio between the association of a given SNP with suicide attempt, and the association of that same SNP with the exposure (e.g., cannabis). The Wald estimate was calculated for all selected SNPs; then we meta-analyzed all Wald estimates using multiplicative random-effect inverse-variance weighted (IVW) meta-analysis (primary analysis). The degree of heterogeneity across the meta-analyzed SNPs informs about the presence of pleiotropy (Fig. 1), which can be quantified using the $Q$ statistic (a significant test suggests presence of pleiotropy). Pleiotropy does not bias the analysis, except in case it is unbalanced. Therefore, to determine whether pleiotropy was unbalanced, we tested the significance of the MR-Egger intercept. A significant test indicates presence of unbalanced pleiotropy, which suggests the need to validate the results using sensitivity analysis methods (see below).

\section{Reverse causation}

To test for reverse causation, we estimated the association of suicide attempt with cannabis, alcohol, and tobacco smoking using the same procedure described above.

\section{Sensitivity analyses}

We performed a series of sensitivity analyses to test the robustness of our findings by reestimating our associations using alternative estimators that make different assumptions on the extent of horizontal pleiotropy: (1) MR-Egger regression, which relaxes the Mendelian randomization assumptions allowing for unbalanced pleiotropy; this test suffers from low power. (2) Weighted median regression, which assumes that at least $50 \%$ of the total weights of the instruments comes from valid instruments. (3) Robust Adjusted Profile Score (RAPS) [29], an estimator that deals with weak instruments and is robust to pleiotropic effects. The main criteria for these sensitivity analyses was to assess whether the direction and the size of the associations were consistent with those obtained using the IVW estimator, which would support the validity of the primary analysis.

\section{Outliers}

We investigated the presence of outliers (i.e., SNP instruments that were driving all the association) using two approaches: (1) leave-one-out analysis, in which associations were re-estimated removing one SNP at the time, and (2) MR Pleiotropy Residual Sum and Outlier (MR-PRESSO) procedure, which performs a correction for outliers possibly reflecting bias due to pleiotropy. Outlier SNPs were removed.

\section{Multivariate Mendelian randomization}

We used multivariable Mendelian randomization [30] to simultaneously estimate associations of all substance use phenotypes on suicide attempt, thus accounting for the correlation among substances. While the univariable Mendelian randomization provides an estimate of the total effect, multivariable Mendelian randomization provides an estimate of the direct effect of each substance on suicide attempt. Therefore, a significant association for a given 
substance would suggest that the effect of this substance on suicide attempt is not explained by other substances (e.g., tobacco smoking has an effect on suicide attempt independently from the effect of tobacco smoking on cannabis use). Conversely, a nonsignificant effect would suggest that the effect of a substance is explained by a jointly exposure to other substances (e.g., the effect of tobacco smoking on suicide attempt is explained by the fact that tobacco smoking also increases the likelihood of cannabis use, which in turn increases the risk of suicide attempt). A detailed description of multivariable Mendelian randomization can be found elsewhere [30, 31]. We included in this analysis substances for which we found evidence of association during the previous steps.

\section{Results}

\section{Identification of instrument SNPs}

From the cannabis, alcohol and smoking GWASs, we identified the following instrument SNPs: 42 SNPs associated with cannabis use $\left(P<1 \times 10^{-5}\right), 53$ SNPs associated with number of drinks per week $\left(P<5 \times 10^{-8}\right), 156$ SNPs associated with smoking initiation $\left(P<5 \times 10^{-8}\right)$, and 28 SNPs associated with smoking heaviness $\left(P<5 \times 10^{-8}\right)$. These were used as instruments to test the association between each substance and suicide attempt. In addition, we identified 21 SNPs associated with suicide attempt $(P<1 \times$ $10^{-5}$ ) for the reverse causation analysis. All the identified SNPs were strong instruments according to the $F$ statistic (Supplementary Table S1).

\section{Association of cannabis use, alcohol use, and tobacco smoking with suicide attempt}

We found evidence for an association of cannabis use with suicide attempt (Table 2 and Fig. 2), with an odds ratio (OR) for this association of 1.18, 95\% confidence intervals (CI) 1.01-1.37 $(P=0.032)$. As shown in Supplementary Table S2, both the $Q$ statistic and the MR-Egger intercept were statistically significant $(P=0.038$ and $P=0.014$, respectively), suggesting presence of unbalanced horizontal pleiotropy. To test whether this was introducing a bias in the analyses, we evaluated the MR-Egger regression sensitivity analysis, which accounts for unbalanced pleiotropy. We found that this analysis was consistent with the primary analyses, and still showed evidence for a contribution of cannabis use on suicide attempt (OR, 2.06; CI, 1.31-3.23; $P=0.003$ ). In addition, both the direction and size of the association found in other sensitivity analysis methods, such as RAPS (OR, 1.21; CI, 1.03-1.42; $P=0.022$ ) and weighted median (OR, 1.34; CI, 1.11-1.62; $P=0.003$ ), were consistent with the primary analysis. We did not detect any significant outlier using the MR-PRESSO and leaveone-out procedures (Supplementary file).

For alcohol use, we found evidence for an increased risk of suicide attempt associated with the use of alcohol, with OR 1.95 (CI, 1.15-3.32; $P=0.013$ ). For this analysis, the heterogeneity $Q$ test was significant $(P=0.013$; Supplementary Table S2), but no evidence of unbalanced pleiotropy was found (MR-Egger intercept, $P=0.335$ ). Furthermore, we found that this result was not influenced by outliers, and that sensitivity analyses yielded consistent results (Table 2).

We also found evidence for an association of smoking initiation with suicide attempt (OR, 1.90; CI, 1.54-2.34; $P<0.001$ ). As for alcohol use, the $Q$ statistic indicates significant heterogeneity $(P<0.001 ;$ Supplementary Table S2), but we found no evidence for unbalanced horizontal pleiotropy (MR-Egger intercept, $P=0.319$ ) and outliers. Overall, consistent estimates in the direction and size of the association were obtained in sensitivity analyses (Table 2). Moreover, we found consistent results for the association of smoking heaviness with suicide attempt (OR, 2.13; CI, 1.13-3.99; $P=0.019$ ), after excluding 1 outlier SNP (rs 10519203; Supplementary file) detected using the leave-one-out analysis. For this analysis, the heterogeneity test indicated presence of significant pleiotropy ( $Q$ statistics, $P=0.001$ ), but there was no evidence of unbalanced horizontal pleiotropy (MR-Egger intercept, $P=0.973)$.

\section{Association of suicide attempt with cannabis use, alcohol use, and tobacco smoking (reverse causation)}

To investigate the possibility of reverse causation, we tested the association of suicide attempt with the four substance use phenotypes. We found that the associations of suicide attempt with cannabis use (OR, 1.02; CI, $0.97-1.07 ; P=0.483)$, alcohol use $(\beta, 0.01 ; \mathrm{CI},-0.01$ to $0.02 ; P=0.232$ ), and tobacco smoking (initiation: $\mathrm{OR}$, 1.02; CI, 0.99-1.04; $P=0.144$; heaviness: $\beta,-0.01$; CI, -0.02 to $0.01 ; P=0.601$ ) were not significant in both primary and sensitivity analyses, thus not supporting reverse causation (Table 2). We found evidence for significant heterogeneity for all phenotypes except for cannabis use, but no evidence was found of unbalanced horizontal pleiotropy for cannabis and tobacco smoking (Supplementary Table S2). Although, for alcohol use, we found evidence for both unbalanced horizontal pleiotropy (MR-Egger intercept, $P=0.019$ ) and for 1 outlier SNP (rs2916138), sensitivity analyses and removing the outlier SNP $(\beta, 0.004 ; \mathrm{CI},-0.01$ to $0.01 ; P=0.489)$ consistently showed no significant associations. 
Table 2 Mendelian randomization estimates for the association of substance use with suicide attempt, and for the association of suicide attempt with substance use.

\begin{tabular}{|c|c|c|c|c|c|c|}
\hline & \multicolumn{3}{|c|}{$\begin{array}{l}\text { Association of substance use with } \\
\text { suicide attempt }\end{array}$} & \multicolumn{3}{|c|}{$\begin{array}{l}\text { Association of suicide attempt with } \\
\text { substance use (reverse causation) }\end{array}$} \\
\hline & No. SNPs & OR $(95 \% \mathrm{CI})$ & $P$ & No. SNPs & $\mathrm{OR} / \beta(95 \% \mathrm{CI})$ & $P$ \\
\hline \multicolumn{7}{|l|}{ Cannabis use } \\
\hline $\begin{array}{l}\text { Inverse variance } \\
\text { weighted }\end{array}$ & 42 & $1.18(1.01-1.37)$ & 0.032 & 21 & $1.02(0.97-1.07)$ & 0.483 \\
\hline $\begin{array}{l}\text { Robust adjusted } \\
\text { profile score }\end{array}$ & 42 & $1.21(1.03-1.42)$ & 0.022 & 21 & $1.01(0.96-1.06)$ & 0.742 \\
\hline Weighted median & 42 & $1.34(1.11-1.62)$ & 0.003 & 21 & $0.99(0.93-1.06)$ & 0.837 \\
\hline MR-Egger (SIMEX) & 42 & $2.06(1.31-3.23)$ & 0.003 & 21 & $1.10(0.95-1.28)$ & 0.186 \\
\hline \multicolumn{7}{|l|}{ Alcohol use (drinks/week) ${ }^{\mathrm{a}}$} \\
\hline $\begin{array}{l}\text { Inverse variance } \\
\text { weighted }\end{array}$ & 53 & $1.95(1.15-3.32)$ & 0.013 & 21 & $0.01(-0.01 ; 0.02)$ & 0.234 \\
\hline $\begin{array}{l}\text { Robust adjusted } \\
\text { profile score }\end{array}$ & 53 & $1.89(1.11-3.23)$ & 0.019 & 21 & $0.00(-0.01 ; 0.02)$ & 0.594 \\
\hline Weighted median & 53 & $1.71(0.78-3.72)$ & 0.179 & 21 & $0.00(-0.01 ; 0.02)$ & 0.541 \\
\hline MR-Egger $\left(\right.$ SIMEX) ${ }^{\mathrm{c}}$ & 53 & $1.72(0.65-4.57)$ & 0.284 & 21 & $0.02(-0.02 ; 0.06)$ & 0.196 \\
\hline \multicolumn{7}{|l|}{ Tobacco smoking initiation } \\
\hline $\begin{array}{l}\text { Inverse variance } \\
\text { weighted }\end{array}$ & 156 & $1.90(1.54-2.34)$ & $<0.001$ & 21 & $1.02(0.99-1.04)$ & 0.144 \\
\hline $\begin{array}{l}\text { Robust adjusted } \\
\text { profile score }\end{array}$ & 156 & $2.00(1.60-2.49)$ & $<0.001$ & 21 & $1.01(0.99-1.02)$ & 0.450 \\
\hline Weighted median & 156 & $2.11(1.59-2.80)$ & $<0.001$ & 21 & $1.01(0.99-1.02)$ & 0.440 \\
\hline MR-Egger (SIMEX) & $155^{\mathrm{b}}$ & $1.20(0.43-3.31)$ & 0.732 & 21 & $0.98(0.95-1.01)$ & 0.123 \\
\hline \multicolumn{7}{|l|}{ Smoking heaviness ${ }^{\mathrm{a}}$} \\
\hline $\begin{array}{l}\text { Inverse variance } \\
\text { weighted }\end{array}$ & 27 & $2.13(1.13-3.99)$ & 0.019 & 21 & $-0.01(-0.02 ; 0.01)$ & 0.601 \\
\hline $\begin{array}{l}\text { Robust adjusted } \\
\text { profile score }\end{array}$ & 27 & $2.19(1.13-4.25)$ & 0.020 & 21 & $-0.01(-0.02 ; 0.01)$ & 0.274 \\
\hline Weighted median & 27 & $2.54(1.21-5.33)$ & 0.014 & 21 & $-0.01(-0.03 ; 0.01)$ & 0.358 \\
\hline MR-Egger (SIMEX) & 27 & $2.23(0.07-66.87)$ & 0.646 & 21 & $0.02(-0.02 ; 0.06)$ & 0.399 \\
\hline
\end{tabular}

The table reports the results for the Mendelian randomization analyses. For each exposure considered (i.e., cannabis use, alcohol use, smoking initiation, and smoking heaviness), we reported the estimate obtained using the inverse-variance weighted method (primary analysis) as well as the estimates obtained with the sensitivity analysis methods. MR-Egger is based on the "no measurement error in the SNP-exposure effects (NOME) assumption". We evaluated this assumption by computing the regression dilution $I_{(\mathrm{GX})}^{2}$ statistic [44]. In case of values below $90 \%$ (suggesting violation of the NOME assumption), simulation extrapolation (SIMEX) correction was applied [45]. $I_{(\mathrm{GX})}^{2}$ for the association of cannabis, alcohol, smoking initiation, smoking heaviness was, respectively, 0.54, 0.97, 0.67, and $0.63 . I_{(\mathrm{GX})}^{2}$ for the association of suicide attempt with substance use phenotypes was 0.74 .

SNP single nucleotide polymorphism, $O R$ odds ratio, $C I$ confidence interval.

${ }^{a}$ Associations are expressed as beta coefficients for the reverse association.

${ }^{\mathrm{b}}$ One outlier SNP was excluded.

${ }^{\mathrm{c}}$ SIMEX correction was applied only to the analysis on the association of suicide attempt with alcohol use.

\section{Testing for direct associations}

Accounting for the correlation among substances using multivariable Mendelian randomization showed that only cannabis use was directly associated with increased risk of suicide attempt, while no direct associations were found for alcohol use and smoking initiation (Table 3). Results were consistent between primary (IVW) and sensitivity (MR-Egger) analyses.

\section{Discussion}

\section{Main findings}

To our knowledge, this is the first study testing the associations of cannabis, alcohol, and tobacco smoking on suicide attempt using Mendelian randomization, a quasiexperimental approach strengthening causal inference. We 

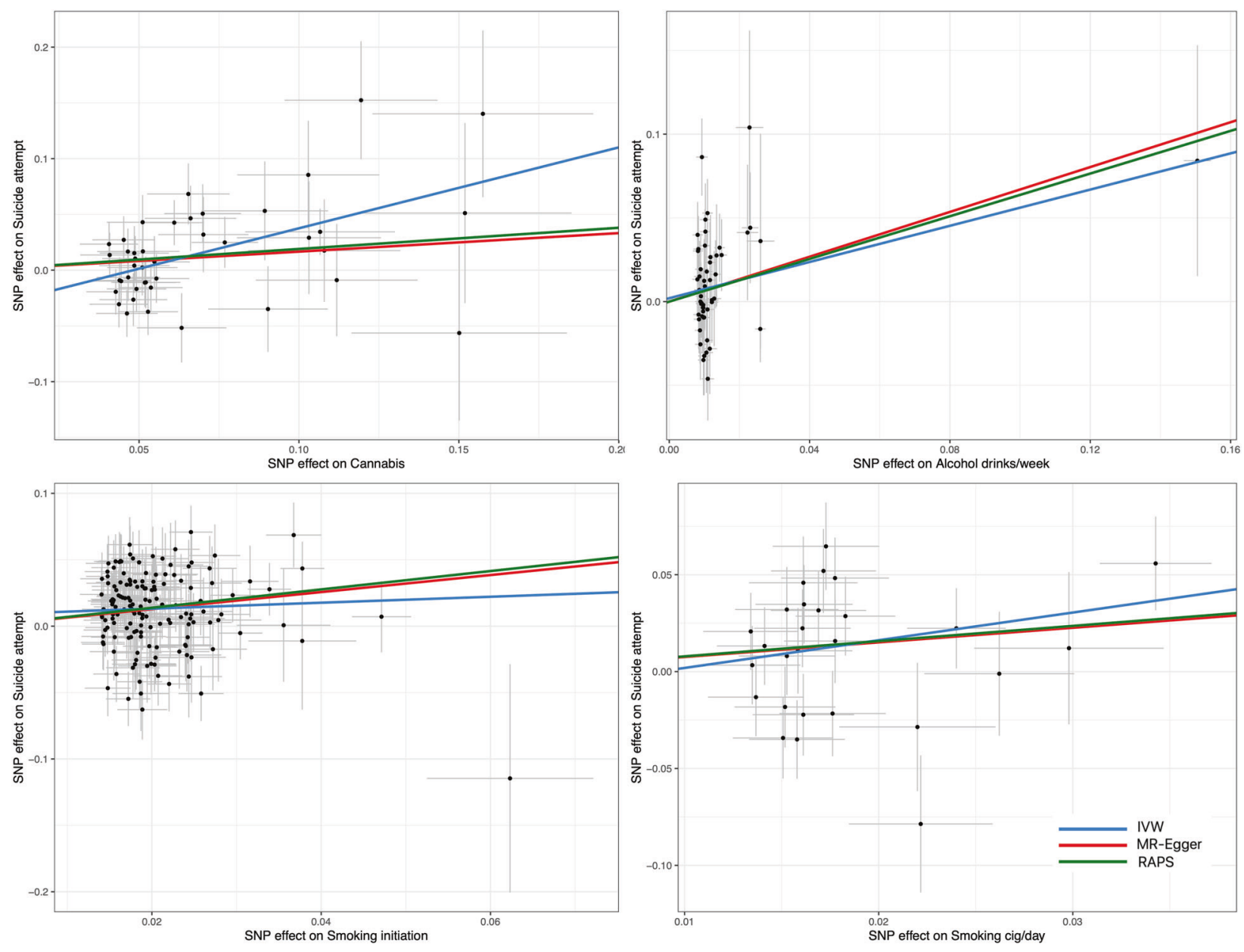

Fig. 2 Mendelian randomization scatter plots for the associations of cannabis, alcohol, and tobacco smoking with suicide attempt. The scatter plots show the associations between the instrument singlenucleotide polymorphisms (SNPs) and the exposure (cannabis, alcohol, tobacco smoking) in the $x$-axis, and the associations between these same SNPs and the outcome (suicide attempt) in the $y$-axis. Lines

represent the regression of the SNP-exposure estimates on the SNPoutcome estimates, which correspond to the meta-analysis of all Mendelian randomization Wald estimates for each single instrument SNP computed using multiplicative random-effect inverse-variance weighted (IVW, red), MR-Egger (blue), and Robust Adjusted Profile Score (RAPS; green) methods.

Table 3 Multivariate Mendelian randomization estimates for the association of substance use with suicide attempt.

\begin{tabular}{|c|c|c|c|c|c|}
\hline & \multicolumn{2}{|c|}{ Inverse variance weighted } & \multicolumn{3}{|l|}{ MR-Egger method } \\
\hline & OR $(95 \% \mathrm{CI})$ & $P$ & OR $(95 \% \mathrm{CI})$ & $P$ & $P$ intercept \\
\hline Cannabis use & $1.25(1.09-1.43)$ & 0.001 & $1.23(1.01-1.50)$ & 0.044 & 0.841 \\
\hline Alcohol use (drinks/week) & $0.90(0.54-1.52)$ & 0.702 & $1.05(0.52-2.10)$ & 0.890 & 0.519 \\
\hline Smoking initiation & $0.96(0.76-1.21)$ & 0.735 & $0.91(0.60-1.40)$ & 0.675 & 0.780 \\
\hline
\end{tabular}

No. of SNPs used in the analysis is 248. $Q$ (245 degrees of freedom), 397.9, $P<0.001$.

found evidence supporting a possible causal role of cannabis, alcohol, and tobacco smoking on suicide attempt. The size of these associations were small-to-moderate, with OR ranging from 1.18 to 1.95 . We found that cannabis use was linked to suicide attempt via a direct pathway, while absence of direct associations for alcohol and smoking suggested that their effect is explained (i.e., mediated) by the other substance use phenotypes. Finally, we found no evidence supporting reverse causation, i.e., the contribution of suicide attempt on cannabis, alcohol and tobacco smoking.

\section{Interpretation of the findings within the existing literature}

Our findings are consistent with previous observational studies showing increased risk of suicide attempt for individual reporting substance use [6-14]. Concerning cannabis 
use, previous meta-analyses showed associations of cannabis use with suicide risk in both youth [10] and adult populations [34], which is in line with our Mendelian randomization findings. Important for triangulation of evidence, our findings are also in line with quasi-experimental studies showing increased risk of suicide attempt associated with cannabis consumption using monozygotic twins discordant for cannabis use [24, 35]. A previous study also suggested stronger associations with suicide risk for heavy cannabis users than for low/moderate cannabis users [36]. However, we could not test for dose-response associations of the frequency/intensity of cannabis use, because this was not measured in the cannabis GWAS. Future quasiexperimental studies should clarify the patterns of substance use that are most likely to increase suicide risk. In addition, although significant, the OR for cannabis use was low, which calls for a cautious interpretation of this finding. Concerning alcohol use and tobacco smoking, our findings are also in line with several previous observational studies reporting associations of alcohol consumption and tobacco smoking with suicide-related outcomes [6-9, 11-13]. However, to our knowledge, no previous study used quasiexperimental designs to test these associations. Such studies are needed to triangulate our results and improve confidence on the causal nature of these associations [15]. For tobacco smoking, we found evidence of association for both smoking initiation and smoking heaviness, which is consistent with previous evidence showing dose-response associations between tobacco use and suicide attempt, and further supporting a causal interpretation of the role of smoking on suicide attempt [12].

Our findings are not consistent with a reverse causation hypothesis (i.e., association of suicide attempt with substance use). However, comparison with the literature is challenging for three main reasons. First, prior developmental studies providing evidence for reverse association assessed suicidal ideation and self-reported suicide attempt [17-19]. These phenotypes are substantially different from the one measured in the suicide attempt GWAS used in the present study (i.e., hospital records for suicide attempt). Second, these studies focused on adolescents/young adults [17-19] while the GWAS samples used for our study covered a wider age group. This is important because mechanisms linking substance use and suicide-related outcomes may vary across the lifespan [37], and examining the joint development of substance use and suicidal behavior remains a key methodological element that is not taken into account in the present study. Third, such prior studies are based on observational data from single samples typically not testing for reverse causation; to our knowledge, no quasi-experimental study or meta-analysis testing reverse causation in the association between suicide-related outcomes and substance use have been published. Therefore, future quasi-experimental studies with adolescents should clarify whether alternative causal pathways are plausible. Although a genetically informed approach such as Mendelian randomization is difficult to apply given the shortage of genotyped pediatric datasets, other designs exploiting information on the relatedness between individuals (e.g., twin or sibling designs) can be used [15].

Several mechanisms may explain the associations of substance use with suicide attempt. First, as shown in quasiexperimental studies, substance use may increase the risk for psychiatric disorders, such as depression and schizophrenia, associated with suicide attempt $[24,35,38-40]$. Quasi-experimental evidence for such associations between substance use and psychiatric disorders were found for cannabis [24, 35], alcohol [40], and tobacco smoking [38, 39]. Second, alcohol use may lead to disinhibition, impulsivity, and impaired judgment [10, 41], thus making individuals more prone to attempt suicide [1]. Third, at the biological level, substance use is associated with inflammatory markers, HPA-axis regulation, serotonin levels, and endocannabinoid system functioning [13, 35, 42], which were all implicated in the neurobiology of mood regulation and suicide [1, 43]. Finally, heavy use of cannabis and alcohol may lead to social problems such as socioeconomic difficulties and disruption of social bonds [5], in turn heightening suicide risk. Clarifying the role of these mechanisms would provide important insights for suicide prevention among substance users. In addition, since our multivariable analysis suggested that only cannabis had a direct effect on suicide attempt, understanding the patterns of association among the different substances is necessary.

\section{Strengths and limitations}

This study uses a robust quasi-experimental approach, based on high-quality GWAS data from international consortia using large samples and a range of sensitivity analyses to support the conclusions. However, the following limitations must be acknowledged. First, our findings are valid under specific assumptions of the instrumental variable approach, including the absence of alternative pathways explaining the associations between instrument SNPs and suicide attempt (horizontal pleiotropy). Second, given the data available, reported associations are not specific to a particular age group. Since both substance use and suicide attempt can have different characteristics in different developmental periods, we cannot exclude alternative patterns of associations and developmental mechanisms according to age groups (e.g., adolescence vs. adult cannabis use). Third, our analyses focused on both sexes combined, as no sex-specific GWASs were available. Fourth, the suicide attempt phenotype was hospital record for suicide attempt, which may include only the most 
serious suicide attempt for which people reached for medical care. Therefore, this may capture only a part of the suicide attempt phenomenon. Fifth, the binary assessment of cannabis use in the lifetime and the unavailability of the heaviness of cannabis use limit the interpretation of the results and call for further investigations.

\section{Conclusions}

In conclusion, this study found evidence for a possible causal contribution of cannabis, alcohol, and tobacco smoking to suicide attempt. This finding triangulates a consistent body of observational and quasi-experimental research on the role of these substances on suicide risk. Considering that cannabis, alcohol, and tobacco are the most commonly used substances, these findings have clear public health implications. Future research aimeing at understanding the role of substance use consuming habits (frequency, quantity, and patterns of association among the different substances) and associated factors are needed to better understand how to prevent suicide among substance users, and to determine limits and feasibility of potential interventions in specific populations (e.g., adolescents, adults, and clinical populations).

\section{Code availability}

Analyses were performed using the $\mathrm{R}$ packages TwoSampleMR [32], Mendelian Randomization [33], and MRPRESSO ( $\mathrm{R}$ version 3.5.2). The computer code is available upon request.

Acknowledgements MO is funded by the European Union's Horizon 2020 research and innovation program (grant agreement no. 793396). GT holds a Canada Research Chair (Tier 1) and a NARSAD Distinguished Investigator Award, and is supported by grants from the Canadian Institute of Health Research (CIHR) (FDN148374 and EGM141899). MCG, JRS, and GT are supported by the Fonds de recherche du Québec-Santé (FRQS) through the Quebec Network on Suicide, Mood Disorders and Related Disorders. MCG receives funding from the American Foundation for Suicide Prevention and holds a Canada Research Chair (Tier 2). NCR and MCG are fellows of the Fonds de Recherche en Santé du Québec. NCR and JRS are supported by grants from the CIHR (PJT-148551) and a CIHRCanadian Center on Substance Use and Addiction Catalyst Grant. JRS is also supported by the Fond Monique Gaumond pour la recherche sur les maladies affectives. RET is funded by the Social Sciences and Humanities Research Council of Canada. Funders have no role in study design, data analysis, interpretation of the data, writing of the paper.

\section{Compliance with ethical standards}

Conflict of interest The authors declare that they have no conflict of interest.
Publisher's note Springer Nature remains neutral with regard to jurisdictional claims in published maps and institutional affiliations.

\section{References}

1. Turecki G, Brent DA. Suicide and suicidal behaviour. Lancet. 2016;387:1227-39.

2. WHO. WHO । Suicide data. WHO. 2015. http://www.who.int/ mental_health/prevention/suicide/suicideprevent/en/. Accessed 24 Feb 2018.

3. Nock MK, Green JG, Hwang I, McLaughlin KA, Sampson NA, Zaslavsky AM, et al. Prevalence, correlates, and treatment of lifetime suicidal behavior among adolescents: results from the National Comorbidity Survey Replication Adolescent Supplement. JAMA Psychiatry. 2013;70:300-10.

4. Goldman-Mellor SJ, Caspi A, Harrington H, Hogan S, Nada-Raja $\mathrm{S}$, Poulton R, et al. Suicide attempt in young people: a signal for long-term health care and social needs. JAMA Psychiatry. 2014; $71: 119-27$.

5. GBD 2016 Alcohol and Drug Use Collaborators. The global burden of disease attributable to alcohol and drug use in 195 countries and territories, 1990-2016: a systematic analysis for the Global Burden of Disease Study 2016. Lancet Psychiatry. 2018; 5:987-1012.

6. Berlin I, Hakes JK, Hu M-C, Covey LS. Tobacco use and suicide attempt: longitudinal analysis with retrospective reports. PLOS ONE. 2015;10:e0122607.

7. Chang HB, Munroe S, Gray K, Porta G, Douaihy A, Marsland A, et al. The role of substance use, smoking, and inflammation in risk for suicidal behavior. J Affect Disord. 2019;243:33-41.

8. Darvishi N, Farhadi M, Haghtalab T, Poorolajal J. Alcohol-related risk of suicidal ideation, suicide attempt, and completed suicide: a meta-analysis. PLoS ONE. 2015;10:e0126870.

9. Flensborg-Madsen T, Knop J, Mortensen EL, Becker U, Sher L, Grønbæk M. Alcohol use disorders increase the risk of completed suicide-irrespective of other psychiatric disorders. A longitudinal cohort study. Psychiatry Res. 2009;167:123-30.

10. Gobbi G, Atkin T, Zytynski T, Wang S, Askari S, Boruff J, et al. Association of Cannabis use in adolescence and risk of depression, anxiety, and suicidality in young adulthood: a systematic review and meta-analysis. JAMA Psychiatry. 2019;76:426-34.

11. Korhonen T, Sihvola E, Latvala A, Dick DM, Pulkkinen L, Nurnberger J, et al. Early-onset tobacco use and suicide-related behavior-a prospective study from adolescence to young adulthood. Addict Behav. 2018;79:32-8.

12. Li D, Yang X, Ge Z, Hao Y, Wang Q, Liu F, et al. Cigarette smoking and risk of completed suicide: a meta-analysis of prospective cohort studies. J Psychiatr Res. 2012;46:1257-66.

13. Malone KM, Waternaux C, Haas GL, Cooper TB, Li S, Mann JJ. Cigarette smoking, suicidal behavior, and serotonin function in major psychiatric disorders. Am J Psychiatry. 2003;160:773-9.

14. Moore TH, Zammit S, Lingford-Hughes A, Barnes TR, Jones PB, Burke M, et al. Cannabis use and risk of psychotic or affective mental health outcomes: a systematic review. Lancet. 2007;370: 319-28.

15. Pingault J-B, O'Reilly PF, Schoeler T, Ploubidis GB, Rijsdijk F, Dudbridge F. Using genetic data to strengthen causal inference in observational research. Nat Rev Genet. 2018;19:566-80.

16. Gilbert R, Widom CS, Browne K, Fergusson D, Webb E, Janson $\mathrm{S}$. Burden and consequences of child maltreatment in high-income countries. Lancet. 2009;373:68-81.

17. Fergusson DM, Horwood LJ, Ridder EM, Beautrais AL. Suicidal behaviour in adolescence and subsequent mental health outcomes in young adulthood. Psychol Med. 2005;35:983-93. 
18. Marschall-Lévesque S, Castellanos-Ryan N, Parent S, Renaud J, Vitaro F, Boivin M, et al. Victimization, suicidal ideation, and alcohol use from age 13 to 15 years: support for the selfmedication model. J Adolesc Health. 2017;60:380-7.

19. Steinhausen H-C, Bösiger R, Metzke CW. Stability, correlates, and outcome of adolescent suicidal risk. J Child Psychol Psychiatry. 2006;47:713-22.

20. Glass TA, Goodman SN, Hernán MA, Samet JM. Causal inference in public health. Annu Rev Public Health. 2013;34:61-75.

21. Burgess S, Scott RA, Timpson NJ, Davey Smith G, Thompson SG. Using published data in Mendelian randomization: a blueprint for efficient identification of causal risk factors. Eur J Epidemiol. 2015;30:543-52.

22. Davies NM, Holmes MV, Davey Smith G. Reading Mendelian randomisation studies: a guide, glossary, and checklist for clinicians. BMJ. 2018;362:k601.

23. Erlangsen A, Appadurai V, Wang Y, Turecki G, Mors O, Werge T, et al. Genetics of suicide attempts in individuals with and without mental disorders: a population-based genome-wide association study. Mol Psychiatry. 2018. https://doi.org/10.1038/ s41380-018-0218-y.

24. Pasman JA, Verweij KJH, Gerring Z, Stringer S, Sanchez-Roige S, Treur JL, et al. GWAS of lifetime cannabis use reveals new risk loci, genetic overlap with psychiatric traits, and a causal influence of schizophrenia. Nat Neurosci. 2018;21:1161.

25. Liu M, Jiang Y, Wedow R, Li Y, Brazel DM, Chen F, et al. Association studies of up to 1.2 million individuals yield new insights into the genetic etiology of tobacco and alcohol use. Nat Genet. 2019;51:237.

26. Wootton RE, Lawn RB, Millard LAC, Davies NM, Taylor AE, Munafò MR, et al. Evaluation of the causal effects between subjective wellbeing and cardiometabolic health: mendelian randomisation study. BMJ. 2018;362:k3788.

27. Choi KW, Chen C-Y, Stein MB, Klimentidis YC, Wang M-J, Koenen $\mathrm{KC}$, et al. Assessment of bidirectional relationships between physical activity and depression among adults: A 2Sample Mendelian Randomization Study. JAMA Psychiatry. 2019. https://doi.org/10.1001/jamapsychiatry.2018.4175.

28. Burgess S, Thompson SG. Avoiding bias from weak instruments in Mendelian randomization studies. Int J Epidemiol. 2011;40:755-64.

29. Zhao Q, Wang J, Hemani G, Bowden J, Small DS. Statistical inference in two-sample summary-data Mendelian randomization using robust adjusted profile score. Math Stat. 2018. arXiv: 180109652

30. Burgess S, Thompson SG. Multivariable Mendelian Randomization: the use of pleiotropic genetic variants to estimate causal effects. Am J Epidemiol. 2015;181:251-60.

31. Sanderson E, Davey Smith G, Windmeijer F, Bowden J. An examination of multivariable Mendelian randomization in the single-sample and two-sample summary data settings. Int J Epidemiol. 2018. https://doi.org/10.1093/ije/dyy262.

32. Hemani G, Zheng J, Elsworth B, Wade KH, Haberland V, Baird $\mathrm{D}$, et al. The MR-Base platform supports systematic causal inference across the human phenome. ELife. 2018;7:e34408.

33. Yavorska OO, Burgess S. Mendelian randomization: an R package for performing Mendelian randomization analyses using summarized data. Int J Epidemiol. 2017;46:1734-9.

34. Borges G, Bagge CL, Orozco R. A literature review and metaanalyses of cannabis use and suicidality. J Affect Disord. 2016; 195:63-74

35. Agrawal A, Nelson EC, Bucholz KK, Tillman R, Grucza RA, Statham DJ, et al. Major depressive disorder, suicidal thoughts and behaviours, and cannabis involvement in discordant twins: a retrospective cohort study. Lancet Psychiatry. 2017;4:706-14.

36. Lynskey MT, Glowinski AL, Todorov AA, Bucholz KK, Madden PAF, Nelson EC, et al. Major depressive disorder, suicidal ideation, and suicide attempt intwins discordant for cannabis dependence and early-onset cannabis use. Arch Gen Psychiatry. 2004;61:1026-32.

37. Staff J, Schulenberg JE, Maslowsky J, Bachman JG, O'Malley PM, Maggs JL, et al. Substance use changes and social role transitions: proximal developmental effects on ongoing trajectories from late adolescence through early adulthood. Dev Psychopathol. 2010;22:917-32.

38. Kendler KS, Neale MC, MacLean CJ, Heath AC, Eaves LJ, Kessler RC. Smoking and major depression. A causal analysis. Arch Gen Psychiatry. 1993;50:36-43.

39. Kendler KS, Lönn SL, Sundquist J, Sundquist K. Smoking and schizophrenia in population cohorts of Swedish women and men: a prospective co-relative control study. Am J Psychiatry. 2015; 172:1092-1100.

40. Fergusson DM, Boden JM, Horwood LJ. Tests of causal links between alcohol abuse or dependence and major depression. Arch Gen Psychiatry. 2009;66:260-6.

41. Pompili M, Serafini G, Innamorati M, Dominici G, Ferracuti S, Kotzalidis GD, et al. Suicidal behavior and alcohol abuse. Int $\mathbf{J}$ Environ Res Public Health. 2010;7:1392-431.

42. Green M, Turner S, Sareen J. Smoking and suicide: biological and social evidence and causal mechanisms. J Epidemiol Community Health. 2017;71:839-40.

43. Vinod KY, Hungund BL. Role of the endocannabinoid system in depression and suicide. Trends Pharmacol Sci. 2006;27:539-45.

44. Bowden J, Davey Smith G, Burgess S. Mendelian randomization with invalid instruments: effect estimation and bias detection through Egger regression. Int J Epidemiol. 2015;44:512-25.

45. Bowden J, Del Greco MF, Minelli C, Davey Smith G, Sheehan NA, Thompson JR. Assessing the suitability of summary data for twosample Mendelian randomization analyses using MR-Egger regression: the role of the I2 statistic. Int J Epidemiol. 2016;45:1961-74. 